\title{
A GIS Based Virtual Urban Simulation Environment
}

\author{
Jialiang $\mathrm{Yao}^{1}$, Hissam Tawfik ${ }^{2}$, and Terrence Fernando ${ }^{1}$ \\ ${ }^{1}$ Future Workspaces Research Centre, University of Salford, \\ Salford, M5 4WT, UK \\ ${ }^{2}$ Information Management \& Communication, Liverpool Hope University College, \\ Liverpool, L16 9JD, UK
}

\begin{abstract}
This paper presents the development of a virtual reality urban planning tool based on GIS and Virtual Environments to facilitate scenario generation and collaborative urban design. Our system provides an open structure for users to support various visualisation and simulation modules, for the prototyping of urban designs. An Urban Scene Generation module is used within this urban planning system for generating the urban layout, on which the various collaborative planning tasks are carried out. A 3D urban model is generated from a GIS database by extracting features such as landscape, roads, and buildings. The system allows the users to place CAD models within the virtual environment. Various statistical data related to population, crime, and employment are also visualised in this virtual environment. A VR based user interface is provided to handle urban simulation data, query information and discuss urban simulation scenarios.
\end{abstract}

Keywords: Urban Planning, Virtual Reality, GIS.

\section{Introduction}

Urban planning is a complex and multidisciplinary process requiring the consideration of a number of economical, sociological, transportation and environmental issues. A successful urban planning project requires the satisfactory combination of cost, environmental and cultural requirements.

It is important at the early urban planning stage, to consider how a plan will affect the urban area [1]. Hence an urban plan, which is simulated and evaluated thoroughly at an early stage, would improve the urban regeneration project in terms of cost, efficiency and productivity. Therefore urban planning is a multi-objective decision making task which aims to plan the urban environment in terms of its physical, social, legal, economical, visual and environmental elements, and could achieve many benefits from systems that can help demonstrate and evaluate various aspects of urban planning at an early stage to key stakeholders and the local community. The adoption of a virtual prototyping design concept can bring decision makers and stakeholders together to exchange ideas, quickly assess alternative and build consensus.

Traditional urban planning uses basic tools, such as 2D-maps and text based documents, to deliver a satisfactory urban design solution to all the stakeholders. More recently, databases, computer graphics, photogrammetry and geographic information system (GIS) technology have been adopted to support the process of 
urban planning [2]. Such tools have proved to be effective for professionals performing urban planning tasks. However, they still lack the capabilities for satisfying the increasing need for collaborative urban planning to allow a wide range of professionals involved in the process to exchange and discuss ideas and problems by visualising and interacting with complex data. The aim of our Collaborative Urban Planning Support System is to provide an intuitive and collaborative urban planning environment to enhance participation of stakeholders in urban planning through the use of innovative IT tools.

\section{Related Work}

Numerous information and communication technologies have been developed to aid the urban planning process in recent years, in response to the need for supporting urban planning through visualisation, urban simulation and public participation.

Chan et al. [3] presented an urban simulation system which combines VR, GIS and database technology, to establish a 3D urban simulation environment (Los Angeles Basin, USA). Bourdakis et al. [4] created a Bath model from 1992 using an industry standard CAD package, AutoCAD, that covers several square kilometres of the historic centre of Bath which is accurate to the sub-metre level. A considerable amount of realism in terms of geometrical representation has been modelled using geometry and texture mapping. City planners have used the model to test the visual impact of a number of proposed developments for the city's future. The Adelaide Model [5] demonstrated that the 3D GIS could provide an effective way to model the urban environment by extruding the third dimension from $2 \mathrm{D}$ maps. Although realistic 3D urban visualisation and simulation make the presentation of ideas and proposals at planning meetings more interesting and understandable for the public, the passive presentation of impressive "realistic" rendering is not enough for scenario simulation which is essential for collaborative urban planning and design [6]. Further research is still needed on the implications of realistic 3D urban models used in different stages of the negotiation and design process [7]. GIS is one of the commonly used methods in urban planning [5,8], but the current systems only provide a way to visualise cities, and do not provide functions for manipulating the models, limiting their use in the collaborative design process.

Some research work also attempted to engage public participation in the planning process at every stage, such as the Woodberry Down Project [9]. It used VRML to deliver urban plans to the public in a visual form, but the feedback collected from the public was still in text form in bulletin boards, which may lose information [2] and cause misunderstandings during the collaborative planning procedure [10].

Very little research has been carried out to develop software environments to support urban planning using emerging technology such as virtual reality, 3D interaction and simulation. Addressing these issues requires the presentation of large and complex urban data types. U-Plan aims to facilitate more natural interaction with these datasets in a VR environment and provides an open architecture to facilitate scenario simulation and evaluation. 


\section{A Virtual Urban Planning Support Environment}

\subsection{Design Requirements}

The aim of our VR Urban Planning Support System is to provide a virtual urban planning environment on which urban planners and stakeholders are able to visualise, manipulate and discuss urban planning issues in real time. This requires the system to have an intuitive interface for multi-user interaction and have the ability to quickly generate urban models and visualise various types of urban planning related datasets such as population, crime, employment and transport. The main user requirements for our system can be summarised as follows:

- Urban models and urban planning scenario generation: The system should have the ability to quickly generate an accurate, detailed and realistic 3D layout of an urban environment on which stakeholders can analyse urban planning issues.

- Effective User Interface: The system should have the ability to interact effectively within virtual environments to perform urban planning analysis, such as performing information query, object creation and removal in order to explore various options.

- Collaborative capability: Multi-user interaction within the virtual environment is needed to support the collaborative urban planning process.

- Supporting different visualisation platforms: The collaborative urban planning environment is required to run on different visualisation platforms, such as a standard workstation, workbench and Reality Room, in order to suit various decision-making and design prototyping purposes.

\subsection{System Architecture}

The proposed system architecture has a software framework that supports distributed interactive visualisation and simulation. The system utilises the Hydra distributed architecture, a microkernel for collaborative visualisation of dynamic simulations. In this environment, the servers are used for generating and managing the urban planning scenarios on which the various collaborative planning tasks and simulation data generation such as traffic network analysis can be carried out. A VR based user interface is provided to handle urban simulation data, query information and urban simulation scenarios. The user interface has been designed to run on a range of displays such as Workbench, Reality Room and standard workstation display (Figure 1).

Hydra is a software framework for distributed, multi-user interactive visualisation and simulation applications. It is designed around a lightweight core that assimilates plugins into the framework at runtime and manages the node layers and shared memory. An API layer enables application developers to interact with these data structures through a system of proxies. These proxies enable Hydra to manage the application requirements against the distribution of services across the network. No specific functionality is defined within the core. Instead, functionality is defined at runtime through the provision of user definable plugins, which play a variety of roles from functional adapters to generic service providers. 
There are several visualisation clients for urban planning and simulation in the system. The client in the client-server architecture, typically, is an application that runs on a PC or workbench and relies on servers to perform some operations, as shown in Figure 2.

A World Manager module [11] is used to manage the Scene graph, viewer for scene graph visualisation and input layer for processing user inputs. The World Manager gets user input from input devices such as a head tracker and Wand which is a hand position tracker with programmable control buttons and passes corresponding data to the Viewer and Task Manager for visual feedback and input processing.

The 3D Widgets Module is a set of libraries, which provide a consistent interface from the desktop VR platform to a workbench. It is necessary to be able to interact with the system on a standard desktop system consisting of a monitor, keyboard, mouse and Spacemouse, to a workbench environment which consisting of headtracker and wand. The Widget also provides cross dimensional support allowing tasks to be completed via 2D and 3D styles of interaction, along with gesture and voice based interaction. To enhance the usability of the system, a combination of WIMP techniques and 3D interaction techniques are supported by 3D Widgets.

This system architecture provides a consistent environment to support development of a distributed visualisation system such that different urban planners can share the same virtual urban scenario at different visualisation platforms and manipulate objects in this virtual urban environment. Further more, the framework provides an open architecture in which system functions can be defined at runtime through the provision of user definable modules.

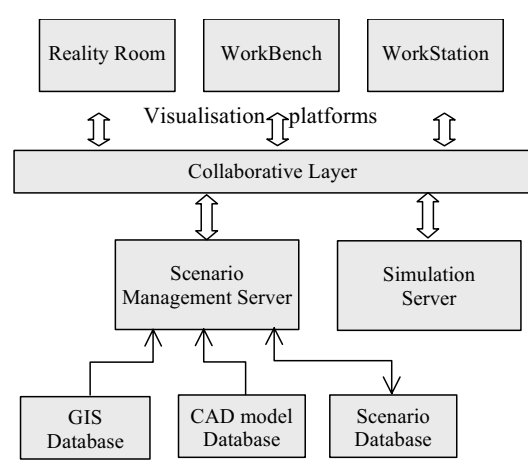

Fig. 1. The system architecture

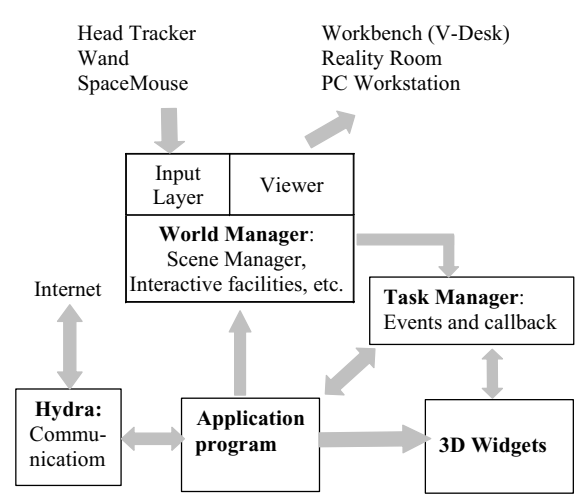

Fig. 2. Client Architecture

\section{GIS Based Urban Model Generation}

The generation of large-scale 3D urban models, using existing 3D modelling tools, such as AutoCAD and Realax, consumes a great deal of time and effort. In this system, ArcGIS is used to manage GIS database, and an urban generation model was developed using $\mathrm{C}++$ and ArcObject to extract geometry and attribute features of the 
landscape, roads, and buildings from ArcGIS database and generate 3D urban models for urban planning in virtual environments.

The 3D landscape model is generated from GIS TIN (Triangular Irregular Network) [12] Layer in which landscape geometry is defined using a group of 3D triangles. Aerial photos are used as texture data to increase the visual realism of the generated landscape.

The 3D Building model is generated according to the building's footprint and height information in the GIS. The building's footprint data is a 2D polygon geometry model [13]. The 3D building model is generated by extruding the polygon according to its height. The building base height is calculated based on its position on the landscape.

The 3D road model is generated using the road central line data from the GIS. A triangle strip generated from the road central line and its width is used to describe the 3D road. The height calculation of road is for the road's triangle strip model, and the method to calculate the height of road is similar to that for buildings.

The 3D urban models use the same coordinate system as that used in GIS. In order to visualise urban models in virtual environments and carry out urban planning, a series of transforms are applied to urban models.

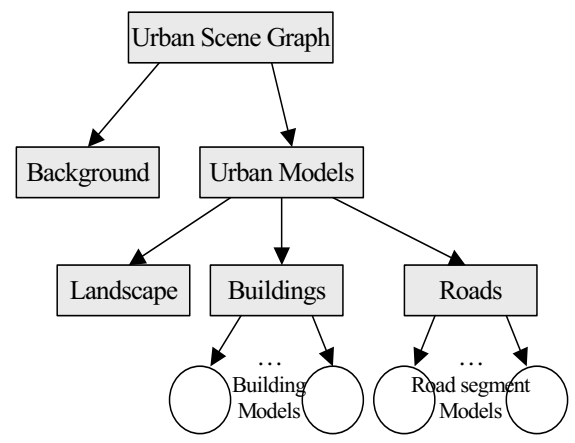

Fig. 3. Urban Scene Graph Organisation

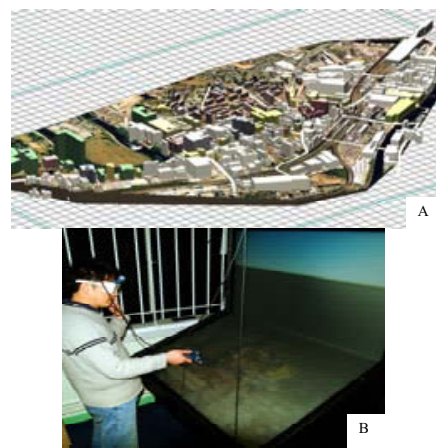

Fig. 4. (a) 3D Urban model and (b) urban model on WorkBench

A tree-like structural organisation method is used to manage and manipulate 3D objects in this system. The main objects include grids for sea-level ground reference, background objects for the surrounding environment and urban models which consists of the landscape, buildings and roads (Figure 3). The ID notations used for the 3D objects within the urban models are identical to their GIS database ID's, making information query of the GIS system straightforward. Figure 4 shows the urban model of Chapel Street in Salford, UK.

The urban models generated from the GIS dataset can be modified through a user interface by adding extra models or removing existing models and saving them as different urban planning scenarios. 


\section{The User Interface}

Based on collaborative urban planning process and a real urban regeneration project, we summarise the requirements for interaction within a virtual environment as follows:

1. Intuitive navigation is vital for collaborative urban planning. Users need the ability to explore any part in the virtual urban scene. This would help users to easily evaluate how a new developing plan affects the surrounding environment, such as how a new tall building affects the skyline of its surroundings and how a newly built supermarket affects the living or working of nearby residents.

2. The ability to create new objects, move and remove existing objects within a virtual urban environment to explore various design options.

3. The ability to mark or highlight an area within the urban environment to focus analysis and discussion around a particular area.

4. The ability to support the user to query information about objects within the urban environment, such as function of a building, highlight particular type of buildings in the area etc.

\subsection{Implementation of Interaction Within Virtual Environments}

Urban Planning scenarios are collaboratively presented on display platforms, where users are provided with series of functions to navigate, manipulate objects in virtual urban environment through interactive user interface.

The implementation of interaction facilities within the virtual environments is based on the world manager, the task manager and the widget manager which provide a consistent interface from the desktop VR to a immersive workbench. The main part of interaction includes system control, navigation and object selection and manipulation.

\section{- System Control}

In order to enable users to carry out necessary operations, the system uses a 3D Widgets Set [14] to provide a 3D form of WIMP-style user interface for the virtual environment. The 3D widgets set provide a series of tools, such as windows, menus (such as cascading menu and cube menu), buttons, text box and scroll list, and are used in virtual environments to facilitate operations such as information query, objects creation, object removal, and information display.

System interaction is conducted through the wand and SpaceMouse input devices. The wand, a tracked hand held device, is used for navigation, object selection, object manipulation and to execute system controls. The SpaceMouse is used for exocentric control of the 3D scene and perform same task as wand. In practice, the wand is more convenient in the Immersive Virtual Environment, while SpaceMouse is the tool used in the standard workstation.

The use of $3 \mathrm{D}$ widgets makes it possible to provide the user with a consistent interface between different platforms, and offers flexibility for users to perform information query, distance measuring, and buildings removal and creation. 
- Navigation

An important aspect in developing the urban planning environment is to provide users with flexible navigational options to explore the urban environment and review urban development plan easily. Two navigational modes are used in this system, namely a world navigation mode and a target-based navigation mode. The World navigation mode, which uses birds-eye view method, is the default navigation mode that is used to allow users to navigate around the entire virtual space. Once an object is selected, the navigation mode will be changed to target based navigation mode (Figure 5), where the selected object will be kept in the centre of the screen.

A virtual compass is provided in this system to aid navigation and object manipulation. It is especially useful when users are unfamiliar with the virtual urban environment.

- Selection and manipulation

Object selection and manipulation are supported through direct interaction using manipulators. The Virtual Ray and virtual hand, which are controlled by input devices are used to conduct direct manipulation operations.

The Virtual Ray is used to select objects, measure distances, mark areas and manipulate widgets as most objects in virtual urban environment are out of reach of users' hands. In the default mode, once the virtual ray hits a selectable object, a bounding box of this object is displayed. If this object is selected using wand or SpaceMouse, the object's colour is changed and system changes its operation mode into Target Based Navigation. When measuring distances, the intersection point of the virtual ray with virtual urban models is traced. A polyline is used to show the path of measured place, and the measured distance is displayed on the toolbar (Figure 6).

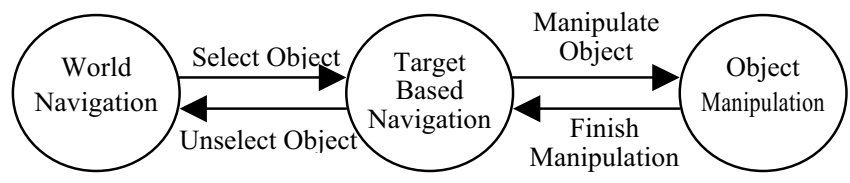

Fig. 5. Operation mode transition during

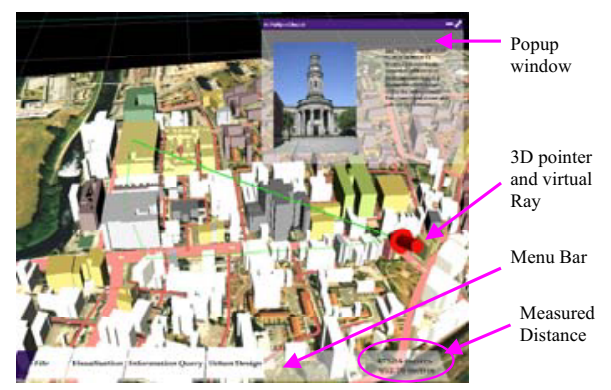

Fig. 6. Information query and distance measureing using virtual ray

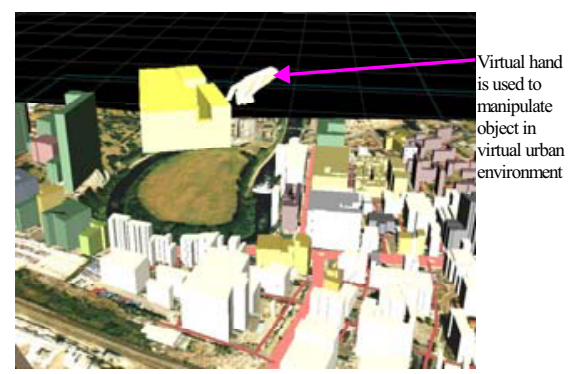

Fig. 7. Object creating, moving using virtual hand 
The virtual hand is used to place objects, such as buildings, at a proper position. The hand's movement and orientation is used to control the object's movement and orientation. In the case of rotating buildings, only one parameter of the orientation the rotation around Y-axis, is used to control the building's orientation. (Figure 7).

\section{Summary}

This work investigates the development of a Collaborative Urban Planning Support System that aims to enhance the urban planning procedure by enabling urban planners to collaboratively make, manipulate and evaluate urban development plans. Urban models are generated using GIS and displayed on Workbench, Reality Room and standard workstation platforms. An intuitive interface using 3D Widgets is provided for users to perform navigation, building manipulation, and information query operations. Initially user evaluation has shown that this system, especially when operating on the Workbench environment, provides an appropriate virtual urban planning platform.

Further development of this system will involve designing an analytical model for traffic network analysis and developing user interfaces suitable for multi-user operation.

\section{Acknowledgement}

This research forms part of the Virtual Urban Laboratory Computer Aided Network (VULCAN) project supported by the Salford local Council. The authors also acknowledge the contribution of S. Bee to the Hydra part and N. Murray to the 3D Widgets part of the system.

\section{References}

1. Jepson, W., Friedman, S.: A Real-Time Visualization System for Large Scale Urban Environments, White Paper (1998)

2. Goodfellow, D.: Collaborative Urban Design through Computer Simulations, A senior honours essay prepared for the School of Urban and Regional Planning, University of Waterloo, Ontario, Canada (1996)

3. Chan, R., Jepson, W., Friedman, S.: Urban Simulation: An Innovative Tool for Interactive Planning and Consensus Building. in Proceedings of the 1998 American Planning Association National Conference, Boston, MA, USA (1998) 43-50

4. Bourdakis, V.: From CAAD to VRML: London Case Study. in The 3rd UK VRSIG Conference Full Paper Proceedings, De Montfort University, UK (1996)

5. Kirby, S.D., Flint, R., Murakami, H., Bamford, E.: The Changing Role of GIS in Urban Planning: The Adelaide Model Case Study. International Journal for Geomatics, 11 (8) (1997) 6-8

6. Pietsch, S., Radford, A., Woodbury, R.: Making and Using a City Model, Adelaide, Australia. in 19th Conference of Education for Computer Aided Architectural Design in Europe, Helsinki, Finland (2001) 442-447 
7. Bourdakis, V.: Making Sense of the City. in CAAD Futures 97, (1997) 663-678

8. Batty, M., Dodge, M., Jiang, B.: GIS and Urban Design. Geographical Information and Planning: European Perspectives (1999) 43 - 65

9. Hudson-Smith, A., Evans, S.: Wired Regeneration: GIS in the third dimension GIS@development (2001)

10. Al-Kodmany, K.: Using Visualization Techniques for Enhancing Public Participation in Planning and Design: Process, Implementation, and Evaluation. Landscape and Urban Planning, 45 (1) (1999) 37-45

11. Marcelino, L., Murray, N., Fernando, T.: A User Interface for Virtual Maintainability in Immersive Environments. in HCI International conference, (2003)

12. Fowler, R.J., Little, J.J.: Automatic extraction of irregular network digital terrain modelsAutomatic extraction of irregular network digital terrain models. Computer Graphics, 13 (2) (1979) 199-207

13. ESRI: Modeling Our World, The ESRI Guide to Geodatabase Design. Environmental Systems Research Institute, Inc. (1999)

14. Murray, N., Goulermas, J.Y., Fernando, T.: Visual Tracking for a Virtual Environment. in HCI International conference, (2003) 